1. An evolutionary advantage for extravagant honesty

2
Seth Bullock

Institute for Complex Systems Simulation,

University of Southampton, UK

sgb@ecs.soton.ac.uk

Tel: +44 (0)2380 595776

Fax: +44 (0)2380 599179 


\title{
An evolutionary advantage for extravagant honesty
}

\author{
Seth Bullock \\ Institute for Complex Systems Simulation, \\ University of Southampton, UK \\ sgb@ecs.soton.ac.uk \\ Tel: +44(0)2380 5957r6 \\ Fax: + 44 (0)2380 599179
}

\begin{abstract}
A game-theoretic model of handicap signalling over a pair of signalling channels is introduced in order to determine when one channel has an evolutionary advantage over the other. The stability conditions for honest handicap signalling are presented for a single channel and are shown to conform with the results of prior handicap signalling models. Evolutionary simulations are then used to show that, for a two-channel system in which honest signalling is possible on both channels, the channel featuring larger advertisements at equilibrium is favoured by evolution.

This result helps to address a significant tension in the handicap principle literature. While the original theory was motivated by the prevalence of extravagant natural signalling, contemporary models have demonstrated that it is the cost associated with deception that stabilises honesty, and that the honest signals exhibited at equilibrium need not be extravagant at all.

The current model suggests that while extravagant and wasteful signals are not required to ensure a signalling system's evolutionary stability, extravagant signalling systems may enjoy an advantage in terms of evolutionary
\end{abstract}


attainability.

Keywords:

Handicap principle, honest signalling, extravagance, evolutionary attainability

\section{Introduction}

Zahavi's handicap principle was proposed as a way of accounting for the evolution of honest signalling by linking the stability of a signalling system to the costs involved in signal production (Zahavi, 1975, 1977). The handicap principle asserts that a signalling system honestly advertising some property (say the quality of a prospective mate, or the hunger of an offspring, or the escape ability of a prey item) will be resistant to invasion by cheats if signalling imposes fitness costs on signallers, and these costs allow signallers with more of the advertised quality to distinguish themselves from those with less by making larger signals (Grafen, 1990a).

This principle was originally inspired by the observation that many natural signals appear needlessly extravagant (Zahavi, 1975, 1977). Peacocks, for example, construct and maintain a tail that is a significant and, to the disinterested observer, irrational drain on resources. Might the same information not be conveyed through a stable signalling system employing much cheaper signals? Similarly, would it not make more sense for stags, stoneflies, manakins, and fireflies to employ discrete and efficient signals in preference to the protracted, exhausting, and potentially dangerous bellowing, drumming, dueting, and flashing that they actually engage in?

A series of game theoretic treatments have shown that signal cost can confer evolutionary stability on handicap signalling systems (e.g., Enquist, 
1985; Grafen, 1990a; Godfray, 1991; Maynard Smith, 1991). However, a subsequent set of treatments have argued that the equilibrium signalling in such models is not "wasteful" and need not handicap signallers (e.g., Bullock, 1997; Getty, 1998, 2006).

In fact, in an early model, Hurd (1995) identifies a scenario within a handicap signalling model in which behaviours that advantage, rather than handicap, signallers can be honest indicators of quality. We can describe his result using the following contrived example. Consider an imaginary arboreal primate. The females of this species are biased in their selection of which males to mate with on the basis of a signal or indicator: whether a prospective mate forages in the highest reaches of the canopy (attractive) or chooses to forage amongst the lower branches (less attractive). Males that reach the highest branches have access to the best of the fruits that they like to eat. Consequently high-quality males, who are light and nimble, would prefer to forage like this even in the absence of any benefit derived from the "signalling component" (Lotem et al., 1999) of their behaviour. However, poor-quality males attempting the same foraging behaviour have a significant chance of falling. As a result, they prefer to forage lower down where there is less risk of falling, even after factoring in the mating opportunities that they are foregoing. At equilibrium, then, foraging behaviour (low or high) is an honest indicator of mate quality (low or high). This signalling system is stabilised by the cost of deceptive signalling (low quality males cannot afford the risks associated with deception), but the (honest) signals that are observed at equilibrium are not costly handicaps, but instead are preferred behaviours that deliver a direct benefit to signallers. 
More generally, it is now understood that whether or not honesty will persist over evolutionary time is determined by the net cost or net benefit associated with a move from honesty to dishonesty (the "marginal net benefit" of honesty), rather than the raw cost of signals made at equilibrium. Consequently, for handicap signalling systems stabilised by the cost of signalling, signallers may produce honest signals of arbitrary raw cost at equilibrium. That is, the space of different handicap signalling systems includes those in which equilibrium signalling behaviour involves signals that impose high gross fitness costs on signallers, but also includes those that impose low costs, zero cost, or even benefits on signallers. Consequently, handicap signalling need not be extravagant in the sense that observed signals are expected to be of (excessively) large magnitude (e.g., Bullock, 1997; Hasson, 1997; Getty, 1998; Bergstrom et al., 2002). For a summary of this modelling literature and a forceful statement of the arguments for reassessing the handicap metaphor, see Hurd \& Enquist (2005) and Getty (2006), respectively.

Here, an alternative account for the evolution of extravagance is considered. Whereas previous game-theoretic models have tended to address the evolutionary stability of honest communication on a single signalling channel, here a model is developed in which the evolution of signalling systems that are able to competitively exclude one another can be explored. The hypothesis to be examined is whether, when considering two signalling systems that both have the potential to be stable and honest, the more extravagant one (i.e, the signalling system employing advertisements of larger magnitude) might enjoy a selective advantage. 


\section{Signalling Over One Channel}

The model follows Grafen (1990a) in taking the form of a simple twoplayer action-response game with continuous traits in which signallers seek to elicit a positive response by advertising some private information that is of interest to receivers. Here, the property being advertised is dubbed "quality", but could be any characteristic of interest to a receiver, including signaller hunger, aggression, escape ability, etc. As such the model is intended to be neutral with respect to many details of the signalling context, including the genetics. If the model were to be refocussed on a specific context, e.g., courtship signalling or offspring begging, it might pay to include factors specific to such a context. As it is, this paper follows Grafen's (1990a) approach in minimising the inclusion of such details in order to achieve generality and simplicity.

Player $S$, a signaller, makes an advertisement with positive perceived magnitude $a \geq 0$ on the basis of a randomly allocated degree of quality, $q$. Player $R$, a receiver or responder, completes the bout of signalling by making a response, $r$, on the basis of $a$ but in ignorance of $q$.

Fitness scores are allocated such that $R$ is rewarded for minimising the difference between the magnitude of its response and the magnitude of signaller quality. ${ }^{1}$

$$
w_{R}=\frac{1}{1+|r-q|}
$$

\footnotetext{
${ }^{1}$ Note that, following Grafen (1990a), receivers are rewarded only for the accuracy of their ability to estimate a signaller's quality, and that over-estimation is treated as equivalent to under-estimation. In reality, there may be situations where the impact of receiver accuracy on fitness varies with signaller quality, and where the fitness consequences of over-estimation differ from those of under-estimation.
} 
Player $S$ gains the benefit $\left(r q^{B}\right)$ of receiving a response, $r$, from Player $R$, but pays the cost $\left(-a q^{C}\right)$ of producing an advert, $a$. In each case the fitness contribution may be mediated by the signaller's own quality, $q$, depending on the values taken by the parameters $B$ and $C$.

$$
w_{S}=r q^{B}-a q^{C}
$$

Where $B$ is positive the impact of receiver response, $r$, on signaller fitness is greater for signallers with higher $q$. Where $B$ is negative, this impact is greater for signallers with lower $q$. Where $B=0$ this impact is independent of signaller quality. Analogously, the value taken by parameter $C$ determines whether the negative fitness impact of advertising is greater for higher quality signallers $(C>0)$ or lower quality signaller $(C<0)$ or is independent of signaller quality $(C=0)$. For example, where $B=0$ and $C=-1$, signallers gain the same benefit from a given receiver response irrespective of their quality, while the cost to a signaller of producing a particular advertisement decreases in direct proportion to signaller quality.

An honest signalling system for this game is a separating equilibrium where signallers produce a unique advertisement, $a$, for each unique value of quality, $q$, being advertised, and receiver response $r$ will equal signaller quality $q$. At the game's non-signalling equilibrium signallers will produce advertisements of zero magnitude for every value of quality being advertised, and receivers will respond with a best guess at signaller quality.

In order to be stable, an honest signalling system must ensure that "better signallers do better by advertising more" (Grafen, 1990a). This condition was formulated by Grafen thus: 


$$
\frac{\partial w_{S} / \partial a}{\partial w_{S} / \partial r} \text { is strictly increasing in } q
$$

For the current model, this yields an inequality, $(B-C) q^{C-B-1}>0$, which is satisfied exclusively by conditions where $B>C$. In such scenarios, any signaller with quality $q$ enjoys an advantage over any competitor with lower quality in terms of the marginal net cost of advertising.

[Figure 1 about here.]

Figure 1 locates this finding within a wider set of models of handicap signalling. For example, the area of figure 1 satisfying the inequality $C<0$ represents Zahavi's $(1975 ; 1977)$ claim that honest signalling will be stable where signalling costs are lower for those signallers with more of the property being advertised. The current model suggests that Zahavi's handicap criterion is neither necessary nor sufficient for the stability of honest signalling. However, the current model is consistent with the results of several subsequent models.

Models addressing the signalling of need have sometimes assumed that the cost of signal production is independent of signaller need, i.e., $C=0$ (e.g., Godfray, 1991; Maynard Smith, 1991). These models have concluded that, in order for such signalling to be honest, the benefits to signallers of observer behaviour must increase with need, i.e., $B>0$ (cf. the heavy vertical arrow in figure 1).

A complementary set of models addressing the signalling of quality have assumed that the benefit to signallers of an observer response is independent of signaller quality, i.e., $B=0$ (e.g., Hurd, 1995). These models have 
concluded that, in order for such signalling to be honest, the cost of signal production must decrease with signaller quality, i.e., $C<0$ (cf. the heavy horizontal arrow in figure 1).

Finally, Grafen's (1990a) result can be represented by the cross-hatched region in figure 1: assuming signaller benefits either increase with quality $(B>0)$ or are independent of it $(B=0)$, Zahavi's constraints on signalling costs $(C<0)$ must hold in order that signalling may be honest. While the current model is consistent with this tightening of Zahavi's claims, the space of stable, honest signalling scenarios defined by Grafen is not coincident with the predictions of the current model. Rather, since the area defined by $B \geq 0$ and $C<0$ is a proper sub-set of the region defined by $B>C$, Grafen's result represents a special case of the current model's findings.

In order to understand how the current model departs from the reasoning of Zahavi, consider the class of scenarios specified by $B>C>0$ (represented by the unhatched shaded region in figure 1). Any signalling channel for which $C>0$ fails to satisfy Zahavi's handicap condition for honest signalling. But where $B>C>0$ the current model predicts that honest signalling will be evolutionarily stable. This class of scenario corresponds to a case in which, say, nestlings are advertising their need by begging. Hungrier nestlings find it more costly to beg than their well-fed competitors $(C>0)$, but this is more than compensated for by the fact that hungrier nestlings stand to benefit more from parental response $(B>C)$. As a consequence, it makes sense for a hungrier chick to beg more than a less needy nestmate even though it costs the hungrier chick more to do so.

By contrast, consider the class of scenarios specified by $B<C<0$ 
(represented by the unshaded hatched region in figure 1). Any signalling channel for which $C<0$ satisfies Zahavi's handicap condition for honest signalling. But where $B<C<0$, the current model predicts that honest signalling will not be evolutionarily stable. Glossed in the same terms as the example above, this class of scenario corresponds to a case in which (for some reason) needier chicks find it less costly to beg than their well-fed nestmates $(C<0)$, but this advantage is extinguished by the fact that they are less able to extract the fitness benefit from parental response $(B<C)$. Perhaps they are not able to metabolise food as efficiently as well-fed chicks (Grafen, 1990a). As a consequence it does not make sense for a hungrier chick to beg more than a less needy nestmate even though it costs the hungrier chick less to do so.

\subsection{Simulation}

In order to explore the attainability of the honest signalling equilibria described in the previous section, the model is translated into a simple simulation. Player $S$, is allocated a degree of quality, $q$, drawn at random from a uniform distribution over the range $\left[q_{\min }, q_{\max }\right]$ and inherits a signalling strategy $\left\langle S_{\alpha}, S_{\beta}\right\rangle$ that defines a mapping, $q \mapsto a$. Similarly, player $R$ inherits a response strategy $\left\langle R_{\alpha}, R_{\beta}\right\rangle$ that defines a mapping, $a \mapsto r$.

During each bout of signalling, $S$ makes an advertisement with positive magnitude $a$ on the basis of $q$,

$$
a=\max \left(0, \operatorname{sgn}\left(S_{\alpha}\right) q^{\left|S_{\alpha}\right|}+S_{\beta}\right) .
$$

$R$ completes the bout of signalling by making a response, $r$, on the basis 
of $a$,

$$
r=\operatorname{sgn}\left(R_{\alpha}\right) a^{\left|R_{\alpha}\right|}+R_{\beta} .
$$

[Figure 2 about here.]

This ensures that, while low-dimensional and smooth, the strategy spaces of $S$ and $R$ comprise a range of mappings from $q$ to $a$ and from $a$ to $r$ that are variously increasing, decreasing, accelerating, decelerating, or flat (see figure 2). Note that as a consequence of the requirement that $a \geq 0$, even where a signalling mapping is not flat, it may be truncated such that either some low- or high-quality signallers make advertisements of zero magnitude. At the conclusion of a bout, scores are allocated to $R$ and $S$ on the basis of equations (1) and (2).

During each simulated generation, each member of a population of $N$ signallers is uniquely paired with a member of a population of $N$ receivers ( $N=1000$ for all results reported here). Each pair engage in a single bout of signalling, after which scores are allocated. Once all pairs have been scored, a new generation of receivers is bred by selecting (with replacement) $N$ parents from the receiver population with probability proportional to their score. Offspring inherit the response strategy of their parent, subject to unbiased mutation in which a perturbation on each strategy component is drawn from the normal distribution with mean zero and standard deviation 0.01 .

A new generation of signallers is bred in a similar fashion. However, since signaller scores may be negative, the probability with which parents are selected from the signaller population is inversely proportional to the rank of their score within the population, rather than proportional to the 
raw score itself. Inherited signaller strategies are mutated in the manner described for response strategies, above.

The new generation of signallers and receivers are then paired, engage in a bout of signalling and bred as before. The simulation is terminated after $G$ generations of this process $(G=5000$ for all results reported here).

Note that, following Grafen (1990b), we model the co-evolution of signaller and receiver strategies without genetic linkage. This allows the model to represent many handicap signalling contexts, but does not realistically capture the genetics when signaller and receiver are related (e.g., parental investment) or signalling is between the sexes of a single species (e.g., courtship signalling).

Before reporting the simulation's behaviour, we will explicitly define what we mean by the term extravagance. A signalling system, $\mathcal{S}$, comprises an equilibrium signalling strategy, $S^{*}$, and the associated equilibrium receiver strategy, $R^{*}$. One signalling system, $\mathcal{S}_{1}$, will be said to be strictly more extravagant than another, $\mathcal{S}_{2}$, if the advertisements made under $\mathcal{S}_{1}$ are of greater magnitude.

$$
\int_{q_{\min }}^{q_{\max }} S_{1}^{*}(q) d q>\int_{q_{\min }}^{q_{\max }} S_{2}^{*}(q) d q
$$

Here, $S_{i}^{*}(q)$ is the magnitude of the advertisement generated by a signaller of quality $q$ using the equilibrium signaller strategy from signalling system $i$.

\section{One Channel: Simulation Results}

First, we corroborate that honest signalling equilibria exist only for scenarios in which $B>C$. For each simulation run, signaller and receiver 
populations were initialised with random strategies, where each element of every player's strategy was drawn from a uniform distribution $[-1,1]$. After a period of simulated coevolution, the resultant signalling behaviour was characterised by two measurements. Receiver prediction error, $\epsilon$, was employed as a proxy for honesty, and signal range, $\rho$, as a proxy for extravagance. ${ }^{2}$

For a particular signalling strategy, the signal range was determined by the signed difference between the magnitude of $a$ when $q=q_{\max }$ and the magnitude of $a$ when $q=q_{\text {min }}$. For each simulated scenario, $\bar{\rho}$ was calculated as

$$
\bar{\rho}=\bar{S}\left(q_{\max }\right)-\bar{S}\left(q_{\min }\right)
$$

Here, $\bar{S}(q)$ is the magnitude of the advertisement generated by a signaller of quality, $q$, employing the mean signaller strategy, $\left\langle\bar{S}_{\alpha}, \bar{S}_{\beta}\right\rangle$. For all results reported here $q_{\min }=1$ and $q_{\max }=5$.

For a particular pair of signaller and response strategies, receiver error was calculated as the mean difference between signaller quality and receiver response across bouts of signalling spanning the range of quality values. For each simulated scenario, $\bar{\epsilon}$ was calculated as

\footnotetext{
${ }^{2}$ Note that (i) the space of signalling strategies used here guarantees that $a$ will always be a monotonic function of $q$, and (ii) we expect that for any honest signalling system $a \approx 0$ for signaller with quality $q=q_{\text {min }}$. This allows us to use the difference between the magnitude of the advertisement given by the lowest and highest quality signallers as a proxy for extravagance. We could also have used the average advertisement magnitude, or calculated the extravagance using equation (6) without qualitatively changing the results reported here. However, the signal range metric employed here has an advantage in that its sign differentiates signallers whose advertisements increase with $q$ from those whose advertisements decrease with $q$, or do not vary with $q$ and are therefore uninformative.
} 


$$
\bar{\epsilon}=\frac{1}{Q} \sum_{j=1}^{Q}\left|\bar{R}\left(\bar{S}\left(q_{j}\right)\right)-q_{j}\right|
$$

Here, $q_{j}$ is drawn from a set of $Q$ values evenly distributed between $q_{\text {min }}$ and $q_{\max }$, and $\bar{R}(a)$ is the magnitude of the response to an advertisement of magnitude $a$ generated by the mean response strategy, $\left\langle\bar{R}_{\alpha}, \bar{R}_{\beta}\right\rangle$.

Note that the stochasticity introduced by mutation ensures that an evolving population will never reach a true equilibrium. We classify a simulation run as having achieved an honest signalling equilibrium where the final receiver population's mean receiver error is below some threshold level, $\bar{\epsilon}<\epsilon_{\text {thresh }}$. (For this to be the case it must also be true that $\bar{\rho} \neq 0$ ). For all results reported here $\epsilon_{\text {thresh }}=0.3$.

[Figure 3 about here.]

First consider stereotypical examples of the evolution of an honest signalling equilibrium and a non-signalling equilibrium, depicted in figure 3 . Solid curves represent the case in which conditions for stable honesty are satisfied $(B>C)$, whereas dashed curves represent the case where these conditions are not satisfied $(B<C)$. In the latter case, both highest quality and lowest quality signallers evolve to produce advertisements with zero magnitude, and receivers evolve to guess signaller quality, achieving a prediction error of $\bar{\epsilon}=0.5$, which is the best that can be achieved in the absence of any information from signallers. Conversely, where $B>C$, highest quality signallers evolve to make advertisements of magnitude approx. 10, while lowest quality signallers again evolve to produce advertisements of approx. zero magnitude, and receivers are able to achieve low response error, $\bar{\epsilon}<\epsilon_{\text {thresh }}$. 
The evolution of the associated strategy parameters is depicted in figure 4 . For $B>C, S_{\alpha}$ and $R_{\alpha}$ stabilise rapidly with the remaining two parameters compensating for one another from around generation 2000. For $B<C$, signallers rapidly evolve negative strategy parameters that guarantee zero magnitude advertisements. Receivers have little selection pressure on their $R_{\alpha}$ value as, in the absence of advertisements, the magnitude of their response is dominated by $R_{\beta}$, which stabilises at a value of around $\left(q_{\min }+q_{\max }\right) / 2$, which is a best guess of signaller quality in the absence of any information from signaller behaviour.

[Figure 4 about here.]

Figure 5 depicts how these measures vary with model parameters $B$ and $C$. Where $B<C$, non-signalling equilibria are achieved: all signallers, irrespective of quality, make uninformative advertisements of zero magnitude $(\bar{\rho} \approx 0)$, and receivers make responses of magnitude $r \approx\left(q_{\max }-q_{\min }\right) / 2$. Conversely, where $B>C$, honest signalling equilibria are always achieved: signallers make honest advertisements such that higher quality signallers employ larger advertisements $(\bar{\rho}>0)$, and receivers are able to recover signaller quality from these advertisements with low error $\left(\bar{\epsilon}<\epsilon_{\text {thresh }}\right)$. Where $B=C$ signalling behaviour repeatedly evolves but is not stable. In summary, simulated populations had no trouble reaching honest signalling equilibria when these equilibria were predicted to exist, and at these equilibria honest signalling behaviour was tightly determined by model parameters such that $\bar{\rho}$ increased exponentially with $B-C$ (see figure 6 ).

[Figure 5 about here.] 


\footnotetext{
${ }^{3}$ Since only one channel may be attended to, this is not a model in which we can explore the evolution of multiple simultaneous signals, either for reasons of increased redundancy or for conveying multiple messages (Johnstone, 1995a, 1996).
}

[Figure 6 about here.]

[Figure 7 about here.]
Note that the absolute values of $B$ and $C$ do not influence the signalling behaviour which is determined by the difference between $B$ and $C$. This means that the model's behaviour does not distinguish between the regions of parameter space depicted in figure 1. For instance, figure 7 shows that scenarios within the region identified by Grafen are equivalent to those in the $B>C>0$ region so long as they share a value for $B-C$.

\section{Signalling Over Two Channels}

Next, we consider what kind of equilibrium signalling behaviour we might expect to evolve where more than one signalling channel exists. When two signalling channels (which may differ in the signalling costs that they impose) are made available to signallers, and receivers must choose which to attend to $^{3}$, can we predict whether one channel will be favoured by evolution, and if so, which?

We extend the current model by including in the expression for signaller fitness a second cost term associated with the additional signalling channel.

$$
w_{S}=r q^{B}-a_{1} q^{C_{1}}-a_{2} q^{C_{2}}
$$

Here, $C_{1}$ and $C_{2}$ are new model parameters that determine the manner in which signaller quality mediates the cost of signalling on channels one and 
two, respectively. Receiver fitness is calculated as before. Following straightforwardly from the single channel case, the evolutionary stability conditions for honesty on each signalling channel are, $B>C_{1}$ and $B>C_{2}$, respectively. Where only one of the channels (or neither) supports stable honest signalling, the question of equilibrium selection is moot. However, if both channels admit stable honest communication (e.g., $B>C_{1}, C_{2}$ ), there exists the possibility that one channel might enjoy an advantage over the other.

Without loss of generality, assume that $C_{1}>C_{2}$. At the outset of any unbiased evolutionary competition between the two evolving signalling systems, the net cost of signalling on channel two must, ceteris paribus, be lowest. Consider that, in such a scenario, on average receivers can be expected initially to treat each channel identically. Hence,

$$
w_{s_{1}}=r q^{B}-a q^{C_{1}}<w_{s_{2}}=r q^{B}-a q^{C_{2}}
$$

In general, where both signalling channels are able to support stable honest signalling (i.e., $B>C_{1}$ and $B>C_{2}$ ), Eq (10) shows that the sign of $C_{1}-C_{2}$ will determine which signalling channel enjoys an initial selective advantage, and the magnitude of $C_{1}-C_{2}$ will determine the extent of this advantage.

\section{Two Channels: Simulation Results}

Here, the original simulation has been augmented such that signallers now inherit a strategy specifying two mappings, $q \mapsto a_{1}$ and $q \mapsto a_{2}$. Likewise, receivers now inherit a mapping for each signalling channel, $a_{1} \mapsto r$ and $a_{2} \mapsto r$, and, in addition, a switch, $\gamma \in\{1,2\}$, that specifies to which channel 
the receiver will exclusively attend. Since this switch element may take only two values, mutation via Gaussian perturbation is inappropriate. Rather, during reproduction, a parental $\gamma$ value is swapped for the alternative allele with mutation probability, $m(m=0.05$ for all results reported here).

Signallers are thus free to employ one, both or neither of the two signalling channels, while receivers are free to develop a different response strategy for each channel, but are constrained to employ one or the other.

[Figure 8 about here.]

Figure 8 depicts the evolutionary change in signaller and receiver behaviour for a scenario where two channels satisfy handicap signalling conditions. On channel $1(C=-2)$, signalling behaviour stabilises after around 2000 generations with $a \approx 35$ for highest quality signallers and $a \approx 0$ for lowest quality signallers. Receivers evolve to pay attention to channel 1 within the first few generations and achieve low response error after 500 generations. By contrast, on channel $2(C=-1.5)$ advertising is rapidly extinguished, and the (unused) receiver strategy (which is under very weak selection pressure) is unable to produce a good estimate of signaller quality.

More generally, the model's parameters $B, C_{1}$ and $C_{2}$ now define a threespace over which we can explore signalling system evolution. In order to visualise the results clearly, figure 9 depicts the model's behaviour over the $C_{1} \times C_{2}$ plane with the third parameter value held constant $(B=0)$. (The model's behaviour is qualitatively similar for other values of $B$, mutatis mutandis.) Since the only difference between channels one and two is captured by the relationship between $C_{1}$ and $C_{2}$, we should expect the panels in figure 9 to exhibit symmetry about $C_{1}=C_{2}$. In addition to this symmetry, 
by comparing the two panels of figure 9 it is apparent that the attainability of honest signalling equilibria increases as either $C_{1}$ or $C_{2}$ fall below $B$, and that in any scenario where both channels admit of an honest signalling equilibrium, whichever channel exhibits advertisements of larger magnitude at equilibrium enjoys an advantage in terms of evolutionary attainability (see figure 10).

[Figure 9 about here.]

[Figure 10 about here.]

\subsection{Competition Between Established Signalling Systems}

[Figure 11 about here.]

Here we simulate abrupt contact between two stable signalling systems that have evolved to equilibrium in isolation. One more extravagant "invading" system for which $C_{1}=-2$ encounters a less extravagant "incumbent" system for which $C_{2}=-1$ (the labels "invading" and "incumbent" are arbitrary and could be reversed).

Initially we allow each system to evolve in isolation as per the rubric of section 2. We fix $B=0$, ensuring that, since $B-C_{1}>B-C_{2}>$ 0 , the equilibrium signalling behaviour in the invading population will be more extravagant than that in the incumbent population, but both signalling systems will be stable and honest.

We then create a new mixed population of $N=1000$ signallers by selecting a random proportion $p$ of individuals from the signaller population of the invading system and combining them with a random proportion $1-p$ of individuals from the signaller population of the incumbent system (the remaining 
signallers are discarded). We construct a mixed receiver population in the same way, with the same ratio of individuals from the two wild-type receiver populations. From this initial condition we simulate a further $G=5000$ generations of evolution.

By varying $p$ we can determine that the more extravagant signalling system enjoys an advantage under these circumstances, being able to achieve fixation (at the expense of the less extravagant signalling system) under a wider range of initial conditions. For the systems depicted in figure 11, the extravagant invading system achieves fixation in the majority of simulation runs when it accounts for only $45 \%$ or more of the initial population. Where the two signalling systems are initially equally represented in the population $(p=0.5)$, the more extravagant system achieves fixation in $90 \%$ of cases.

Despite its nominal disadvantage the weaker signalling system is evolutionarily stable, not only against rare mutants (which is attested to by the results presented in section 3), but also against large numbers of signallers and receivers with strategies that are optimally co-adapted to each other. For both of the systems simulated here, an invading population fully half the size of the incumbent population $\left(p=\frac{1}{3}\right)$ is extremely unlikely to oust the incumbent signalling system.

\section{Discussion}

The model presented here suggests that there are grounds for expecting handicap signalling to appear extravagant - signalling systems employing channels that exhibit signals of larger perceived magnitude at equilibrium are favoured by evolution. It might appear to be consistent with Zahavi's 
(1975; 1977) original arguments that evolution favours the largest and thus most costly signalling system. However, it is more accurate to conclude that, within the space of signalling channels that satisfy handicap signalling criteria, it is those that are cheapest that are advantaged and that this cheapness also results in escalated levels of advertisement magnitude.

Consider two competing signalling channels characterised by $B>C_{1}>$ $C_{2}$. We have seen that while honest signalling is possible on either channel, in general signals will be cheaper on channel 2. Results presented here support two intuitions: first, signallers that employ the cheaper channel will tend to enjoy a selective advantage; second, in order to impose signalling costs of a magnitude sufficient to stabilise signalling on the cheaper channel, greater evolutionary escalation of advertisement magnitude will be required.

How generally should these results be expected to hold? First I will consider issues raised by the implementation of the model as a simulation. Second I will consider constraints on generality due to the form of the model itself.

The initial game theoretic treatment presented in section 2 introduces some theoretical assumptions in the form of game structures and fitness functions. However, the subsequent evolutionary simulation model additionally involves an explicit fitness landscape (i.e., a genetic encoding that imposes a neighbourhood relationship over strategies) and a specific algorithm that moves an explicit, finite population across this landscape, using particular genetic operators and mechanisms for selecting between potential parents. How confident can we be that the way the model behaves can be attributed to the form of the game and its fitness functions, rather than the algorithmic 
devices introduced in order to implement it as an individual-based simulation model? The analytic intractability of simulation models typically prevents a conclusive answer to this question, just as the reliance of a mathematical model on its idealising assumptions (e.g., an infinite population, zero sampling error, differentiable fitness functions) can be hard to assess.

However, the behaviour of the current model is robust to alternative strategy space encodings (e.g., restricting signallers and receivers to linear mappings of the form $a=m q+c$ or $a=q \tan (\theta)+c)$, alternative genetic operators (e.g., a range of mutation operators), alternative selection mechanisms (e.g., local competition between neighbouring members of a population distributed over a two-dimensional rectangular lattice), and alternative initial conditions (e.g., converged on non-signalling equilibrium behaviour).

The relationship between the findings presented here and the more fundamental assumptions made in defining the game itself is less clear and deserves more analysis, particularly as the form of the equations governing key relationships was influenced as much by their simplicity as their realism. The game employs continuous traits where a small change in, say, a signaller's quality or the magnitude of an advertisement results in a small change in the cost associated with making that advertisement. This need not be true of models that employ discrete traits where the notion of cheap signals escalating in magnitude until they achieve evolutionary stability may not hold. The model does not explicitly address the genetics of signalling systems where signallers and receivers may reproduce sexually, or may be related. The game does not include noise on signal production or perception, and does not recognise the difference between the receiver's perception of 
an advertisement's magnitude and that of the signaller. As a consequence of these simplifications, we can expect signallers with minimum quality to make advertisements of zero magnitude. This expectation is unlikely to survive a more sophisticated treatment of the psychophysics of the signaller and receiver roles, e.g., the inclusion of perceptual error, "just noticeable differences" in magnitude and how these scale with the magnitude of a stimulus.

Finally, the current model assumes (along with previous models, e.g., Grafen, 1990a) that receivers are selected for their raw accuracy in estimating signaller quality. The impact on receiver fitness of overestimation is deemed equivalent to that of underestimation, and independent of the true value being advertised. These assumptions seem rather crude when contrasted with the subtle attention paid to signaller fitness, and are unlikely to hold for many natural signalling systems where, for instance, mistakenly fleeing contests with weaklings has very different implications to erroneously fighting much stronger opponents, and passing over first-class suitors differs significantly from bearing the offspring of poor quality mates. Future work will adapt the simulation paradigm employed here to use the outcomes of receiver decision making as a more appropriate proxy for fitness than the raw accuracy of their estimations of signaller quality.

\section{Conclusion}

Zahavi's (1975) estimation that extravagant and exaggerated handicaps are widespread or even endemic within natural signalling systems has proven difficult to assess empirically (see, e.g., Johnstone, 1995b; Kilner \& Johnstone, 1997; Godfray \& Johnstone, 2000; Kotianho, 2001). While the work 
presented here reiterates that natural handicaps need not incur high costs (or indeed, any cost) at equilibrium, it does predict that under some circumstances a handicap signalling system will tend to involve signals of large subjective magnitude. This result might account for our impression of the abundance of extravagance in natural signals - especially if there is significant correlation between our sensory apparatus and that of the receivers for which the signals were evolved.

\section{Acknowledgements}

This paper benefited from conversation with the late John Maynard Smith, Rufus Johnstone, Richard Watson, Jason Noble, James Dyke and the University of Leeds Biosystems group, and from the comments of anonymous reviewers.

\section{References}

Bergstrom, C. T., Számadó, S., \& Lachmann, M. (2002). Separating equilibria in continuous signalling games. Phil. Trans. R. Soc. Lond. B, 357, $1595-1606$.

Bullock, S. (1997). An exploration of signalling behaviour by both analytic and simulation means for both discrete and continuous models. In P. Husbands, \& I. Harvey (Eds.), Proceedings of the Fourth European Conference on Artificial Life (pp. 454-463). Cambridge, MA: MIT Press.

Enquist, M. (1985). Communication during aggressive interactions with particular reference to variation in choice of behaviour. Anim. Behav., 33, $1152-1161$. 
Getty, T. (1998). Handicap signalling: when fecundity and viability do not add up. Anim. Behav., 56, 127-130.

Getty, T. (2006). Sexually selected signals are not similar to sports handicaps. Trends Ecol. Evol., 21, 83-88.

Godfray, H. C. J. (1991). Signalling of need by offspring to their parents. Nature, 352, 328-330.

Godfray, H. C. J., \& Johnstone, R. A. (2000). Begging and bleating: The evolution of parent-offspring signalling. Phil. Trans. R. Soc. Lond. B, 355, $1581-1591$.

Grafen, A. (1990a). Biological signals as handicaps. J. Theor. Biol., 144, $517-546$.

Grafen, A. (1990b). Sexual selection unhandicapped by the Fisher process. J. Theor. Biol., 144, 473-516.

Hasson, O. (1997). Towards a general theory of biological signaling. J. Theor. Biol., 185, 139-156.

Hurd, P. L. (1995). Communication in discrete action-response games. J. Theor. Biol., 174, 217-222.

Hurd, P. L., \& Enquist, M. (2005). A strategic taxonomy of biological communication. Anim. Behav., 70, 1155-1170.

Johnstone, R. A. (1995a). Honest advertisement of multiple qualities using mutiple signals. J. Theor. Biol., 177, 87-94. 
Johnstone, R. A. (1995b). Sexual selection, honest advertisement, and the handicap principle: Reviewing the evidence. Biol. Rev., 70, 1-65.

Johnstone, R. A. (1996). Multiple displays in animal communication: "backup signals" and "multiple messages". Phil. Trans. R. Soc. Lond. B, 352, 329-338.

Kilner, R., \& Johnstone, R. A. (1997). Begging the question: are offspring solicitation behaviours signals of need? Trends Ecol. Evol., 12, 11-15.

Kotianho, J. S. (2001). Costs of sexual traits: a mismatch between theoretical considerations and empirical evidence. Biol. Rev., 76, 365-376.

Lotem, A., Wagner, R. H., \& Balshine-Earn, S. (1999). The overlooked signaling component of nonsignaling behavior. Behav. Ecol., 10, 209-212.

Maynard Smith, J. (1991). Honest signalling: The Philip Sidney game. Anim. Behav., 42, 1034-1035.

Zahavi, A. (1975). Mate selection - A selection for a handicap. J. Theor. Biol., 53, 205-214.

Zahavi, A. (1977). The cost of honesty (further remarks on the handicap principle). J. Theor. Biol., 67, 603-605. 


\section{List of Figures}

1 Schematic representation of the current model's $B \times C$ parameter space, indicating the handicap signalling regime suggested by Zahavi (1975, 1977), $C<0$ (diagonal hatching), and the handicap signalling regimes suggested by models due to Grafen (1990a): $B \geq 0$ and $C<0$ (cross-hatching); Godfray (1991) and Maynard Smith (1991): vertical arrow defined by $B>0$ and $C=0$; and Hurd (1995): horizontal arrow defined by $B=0$ and $C<0)$. The current model predicts that honesty will be stable when $B>C$ (grey shaded region) which is not consistent with Zahavi's original claim, but is consistent with the findings of the subsequent models represented here. . . . . 31

2 Examples of signalling strategies mapping a signaller's private quality, $q$, onto a public advertisement of perceived magnitude a. For all functions depicted, $S_{\beta}=10$, with $S_{\alpha}$ varying from +2 (uppermost) through zero (horizontal) to -2 (lowermost). Note that advertisements may not have negative magnitude. . 32

3 Change in signaller behaviour (upper panel) and receiver behaviour (lower panel) over evolutionary time under conditions that support honest signalling ( $B=0, C=-1$; solid curves) and conditions that do no $(B=0, C=+1$; dashed curves). For signallers, a pair of curves indicate evolutionary change in the magnitude of advertisements $(a)$ given by highest quality and lowest quality signallers employing the mean signaller strategy at each generation during two representative simulation runs. For receivers, each curve indicates evolutionary change in the receiver error $(\bar{\epsilon})$ produced by receivers employing the mean receiver strategy at each generation during the same two simulation runs. The dotted grey line at $\bar{\epsilon}=\epsilon_{\text {thresh }}$ indicates the threshold on receiver error that was used to distinguish the attainment of honest signalling from failure to do so. Parameters: $G=5000, N=1000, q_{\min }=1, q_{\max }=5$, $\epsilon_{\text {thresh }}=0.3, Q=400 \ldots \ldots \ldots \ldots$ 
4 Evolution of signaller and receiver parameters for the two simulation runs depicted in figure 3 . For both cases $B=0$. The upper panel shows the successful evolution of an honest signalling system where $C=-1$. The lower panel shows the evolution of a non-signalling equilibrium where $C=+1$. . . 34

5 Isoclines representing mean signal range $(\bar{\rho})$ in the final generation of simulation runs sampling $17 \times 17$ evenly distributed points in the $B \times C$ parameter space. Each data point represents an average over 25 simulations runs. Where $B<C$, $\bar{\rho} \approx 0$ and $\bar{\epsilon} \approx 0.5$, i.e., non-signalling equilibria were attained. Where $B>C, \bar{\rho}>0$ (increasing exponentially with $B-C$ ) and $\bar{\epsilon} \ll \epsilon_{\text {thresh }}$, i.e., signalling equilibria were attained. Parameters: $G=5000, N=1000, q_{\min }=1, q_{\max }=5, n=25$, $\epsilon_{\text {thresh }}=0.3, Q=400 \ldots \ldots \ldots \ldots$

6 Data from the simulation runs depicted in figure 5 aggregated and re-plotted against $B-C$. The dashed curve shows the proportion of runs achieving an honest signalling equilibrium (i.e., for which $\bar{\epsilon}<\epsilon_{\text {thresh }}$ ). The solid curve shows the average value of $\bar{\rho}$ normalised w.r.t. the maximum observed $\bar{\rho}$ value (std. dev. values were small, varying between $1 \%$ and $3 \%$ of the mean). The inset plot re-plots the solid curve on a log scale. 36

7 Honest signalling system behaviour for ten distinct scenarios for which $B>C$. Ten evolved signaller mappings, $q \mapsto a$, separate into two bundles, one for scenarios where $B-C=1$ (grey curves, $a$ ) and one for scenarios where $B-C=2$ (solid curves, $A$ ). In each case, receiver strategies produce responses, $r$, that are near optimal (i.e., the dashed response curves lie close to the dotted line, $\left.q \mapsto r^{*}\right)$. The two sets of $\{B, C\}$ scenarios depicted are $\{\{-1,-2\},\{0,-1\},\{1,0\},\{2,1\},\{3,2\}\}$ (grey) and $\{\{0,-2\},\{1,-1\},\{2,0\},\{3,1\},\{4,2\}\}$ (black). Simulation parameters are as per figure $5 . \ldots . . . .37$ 
8 Change in signaller behaviour (upper panel) and receiver behaviour (lower panel) over evolutionary time under conditions that support honest signalling on two competing signalling channels $\left(B=0, C_{1}=-2\right.$, solid curves; $B=0, C_{2}=-1.5$, dashed curves). For signallers, a pair of curves for each channel indicate evolutionary change in the magnitude of advertisements $(a)$ given by highest quality and lowest quality signallers employing the mean signaller strategy at each generation during a representative simulation run. For receivers, two curves indicate evolutionary change in the receiver error $(\bar{\epsilon})$ produced by receivers employing the mean receiver strategy for each channel at each generation during the same simulation run. A third curve (grey) indicates the proportion of receivers paying attention to channel 1. The dotted grey line at $\bar{\epsilon}=\epsilon_{\text {thresh }}=0.3$ indicates the threshold on receiver error that was used to distinguish the attainment of honest signalling from failure to do so. Simulation parameters are as per figure 5 plus $m=0.05 \ldots \ldots$. . . . . . . . . . 38

9 Both panels depict results from the same simulation runs sampling $17 \times 17$ evenly distributed points in the $C_{1} \times C_{2}$ parameter space ( $B=0$ in all cases). Each data point represents an average over 25 simulations runs. Upper panel: the frequency with which honest signalling equilibria are discovered on channel 1 (grey isoclines) and channel 2 (heavy isoclines). Lower panel: the signed difference between the mean signal range on each channel at honest signalling equilibrium, $\bar{\rho}_{1}-\bar{\rho}_{2}$. Isoclines are labeled to indicate scenarios for which $\bar{\rho}_{1} \gg \bar{\rho}_{2}, \bar{\rho}_{1}=\bar{\rho}_{2}$, and $\bar{\rho}_{1} \ll \bar{\rho}_{2}$. (This measure is undefined for the upper right quadrant since no honest signalling equilibria were achieved in this region of parameter space). Parameters as figure 8 . . . . . . . 39 
10 The data from the lower left quadrant of figure 9 (i.e., where both $B>C_{1}$ and $B>C_{2}$ ) are aggregated and replotted against $C_{1}-C_{2}$. The solid curve represents the signed difference between the proportion of simulation runs that achieve honest signalling equilibrium on channel 1 and the proportion that achieved honesty on channel 2 . The dashed curve represents $\bar{\rho}_{1}-\bar{\rho}_{2}$, being the signed difference between the average equilibrium signal range on channels 1 and 2 (std. devs. were small, varying being between $1 \%$ and $3 \%$ of the mean). . . . . 40

11 Competition between two established wild-type signalling systems with varying initial frequency in a randomly mixed initial population. The solid curve represents the proportion of $N=20$ simulation runs that fixate on the extravagant invading signalling system $(C=-2)$ after $G=5000$ post-contact generations of evolution. The dashed curve represents the proportion of runs in which the less extravagant incumbent signalling system $(C=-1)$ fixated. $B=0$ for all runs. . . . . . . 41 


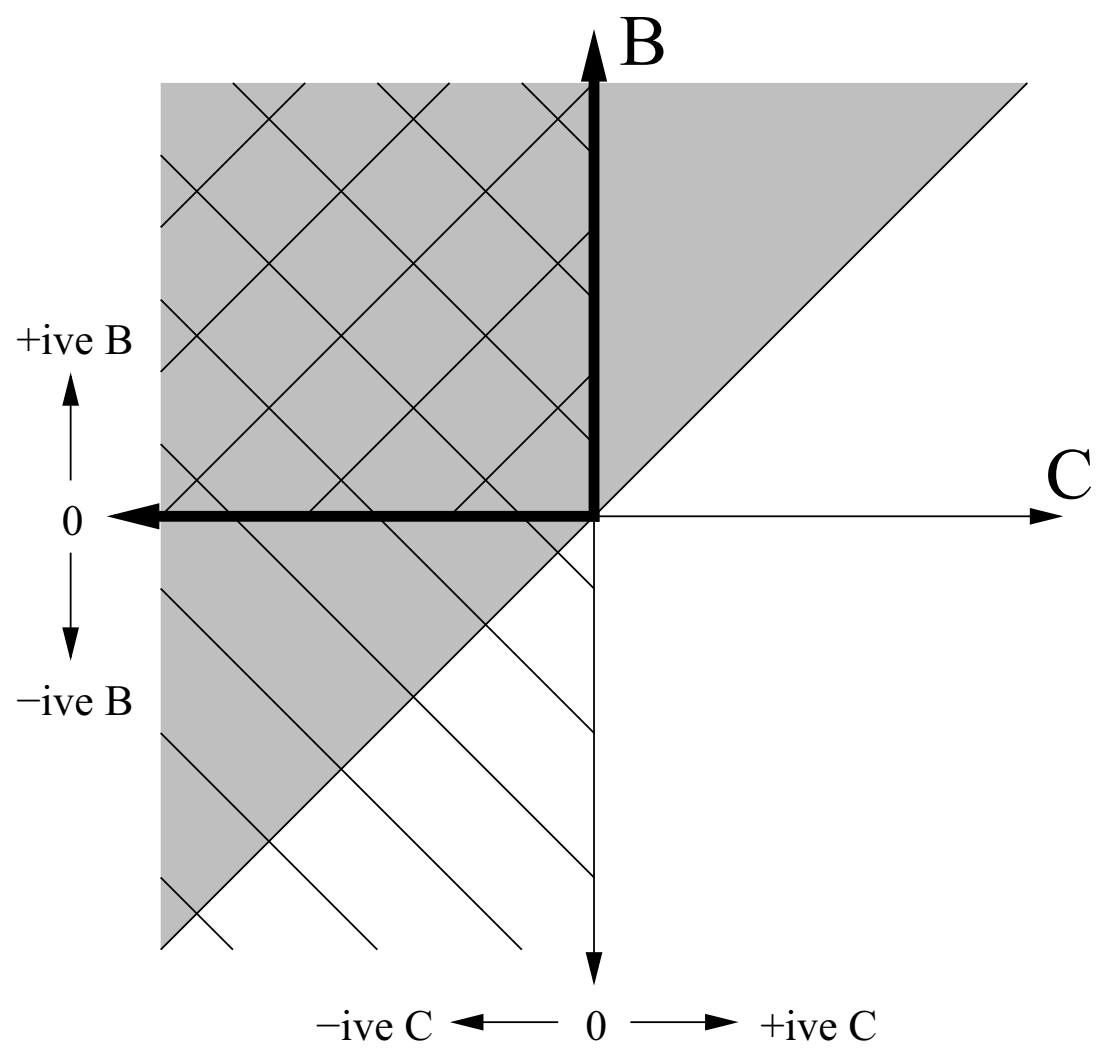

Figure 1: Schematic representation of the current model's $B \times C$ parameter space, indicating the handicap signalling regime suggested by Zahavi $(1975,1977), C<0$ (diagonal hatching), and the handicap signalling regimes suggested by models due to Grafen (1990a): $B \geq 0$ and $C<0$ (cross-hatching); Godfray (1991) and Maynard Smith (1991): vertical arrow defined by $B>0$ and $C=0$; and Hurd (1995): horizontal arrow defined by $B=0$ and $C<0$ ). The current model predicts that honesty will be stable when $B>C$ (grey shaded region) which is not consistent with Zahavi's original claim, but is consistent with the findings of the subsequent models represented here. 


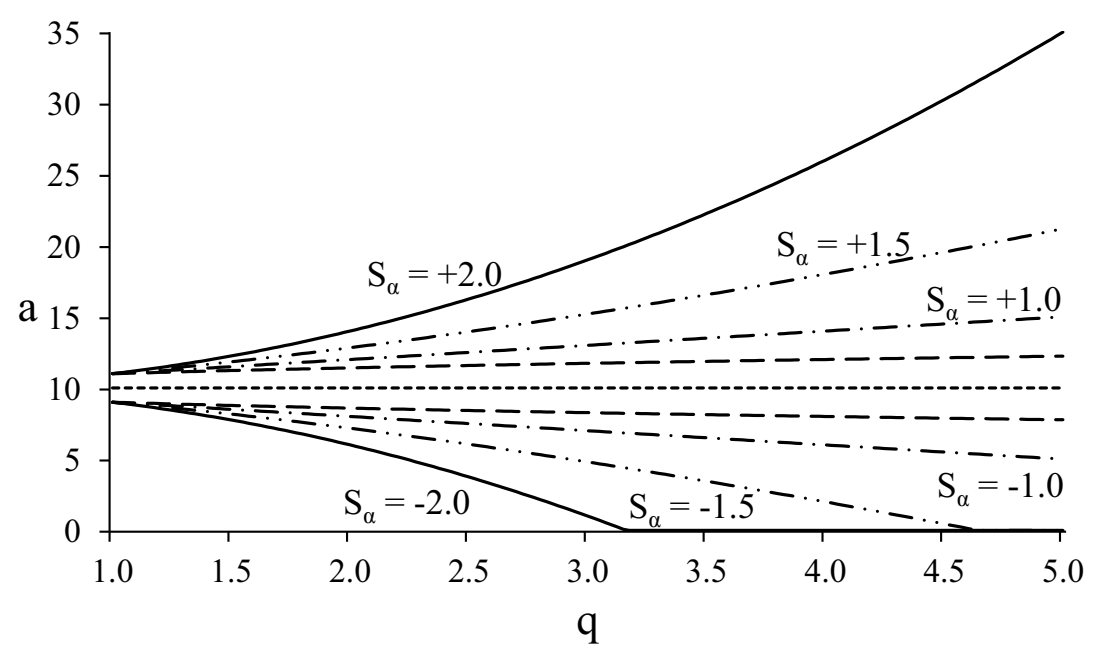

Figure 2: Examples of signalling strategies mapping a signaller's private quality, $q$, onto a public advertisement of perceived magnitude $a$. For all functions depicted, $S_{\beta}=10$, with $S_{\alpha}$ varying from +2 (uppermost) through zero (horizontal) to -2 (lowermost). Note that advertisements may not have negative magnitude. 

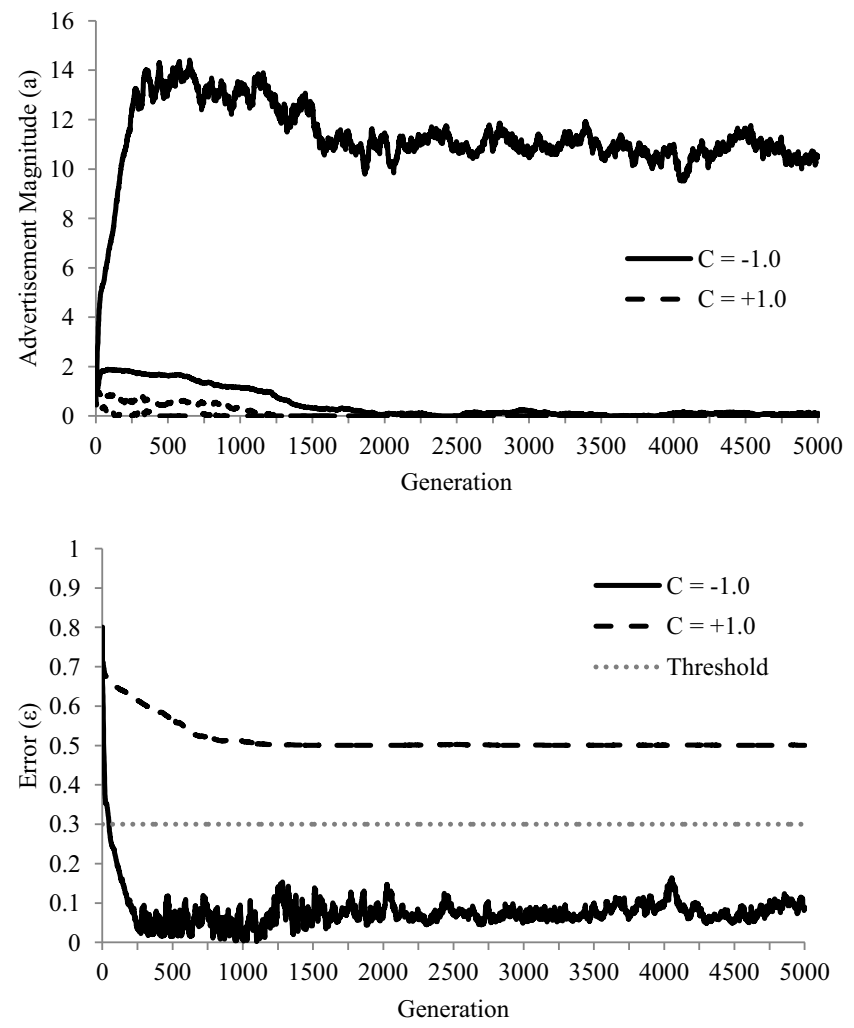

Figure 3: Change in signaller behaviour (upper panel) and receiver behaviour (lower panel) over evolutionary time under conditions that support honest signalling $(B=0, C=-1$; solid curves) and conditions that do no $(B=0, C=+1$; dashed curves). For signallers, a pair of curves indicate evolutionary change in the magnitude of advertisements $(a)$ given by highest quality and lowest quality signallers employing the mean signaller strategy at each generation during two representative simulation runs. For receivers, each curve indicates evolutionary change in the receiver error $(\bar{\epsilon})$ produced by receivers employing the mean receiver strategy at each generation during the same two simulation runs. The dotted grey line at $\bar{\epsilon}=\epsilon_{\text {thresh }}$ indicates the threshold on receiver error that was used to distinguish the attainment of honest signalling from failure to do so. Parameters: $G=5000, N=1000$, $q_{\min }=1, q_{\max }=5, \epsilon_{\text {thresh }}=0.3, Q=400$. 

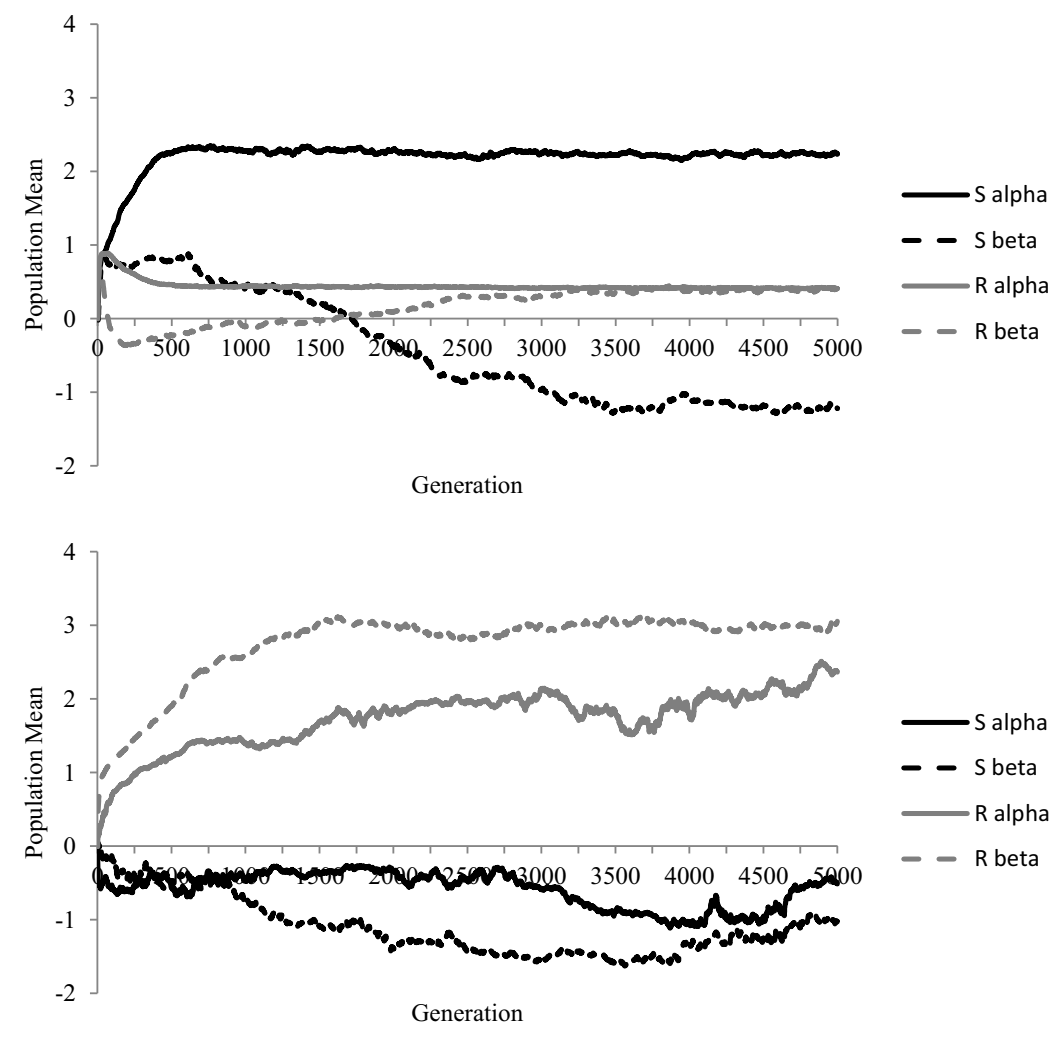

Figure 4: Evolution of signaller and receiver parameters for the two simulation runs depicted in figure 3 . For both cases $B=0$. The upper panel shows the successful evolution of an honest signalling system where $C=-1$. The lower panel shows the evolution of a non-signalling equilibrium where $C=+1$. 


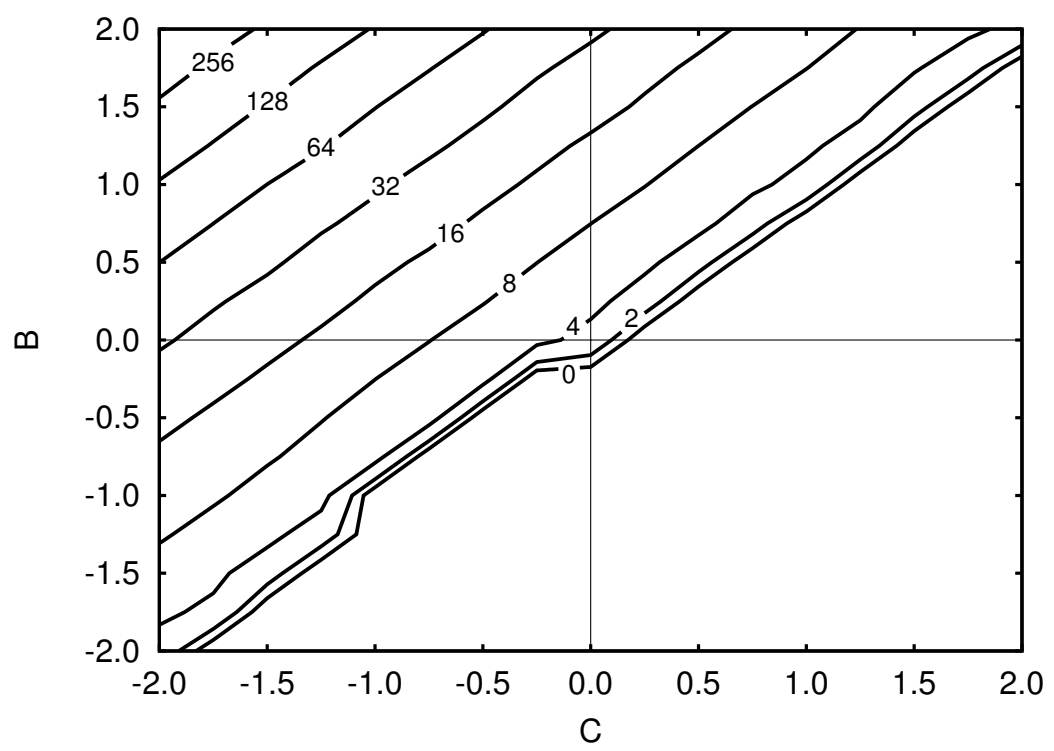

Figure 5: Isoclines representing mean signal range $(\bar{\rho})$ in the final generation of simulation runs sampling $17 \times 17$ evenly distributed points in the $B \times C$ parameter space. Each data point represents an average over 25 simulations runs. Where $B<C, \bar{\rho} \approx 0$ and $\bar{\epsilon} \approx 0.5$, i.e., non-signalling equilibria were attained. Where $B>C, \bar{\rho}>0$ (increasing exponentially with $B-C)$ and $\bar{\epsilon} \ll \epsilon_{t h r e s h}$, i.e., signalling equilibria were attained. Parameters: $G=$ $5000, N=1000, q_{\min }=1, q_{\max }=5, n=25, \epsilon_{\text {thresh }}=0.3, Q=400$. 


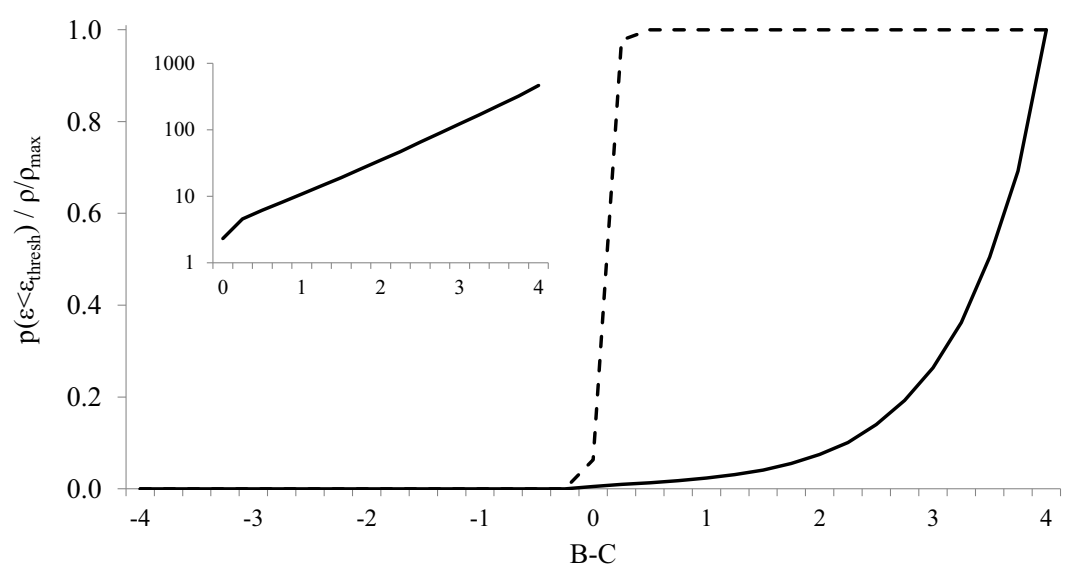

Figure 6: Data from the simulation runs depicted in figure 5 aggregated and re-plotted against $B-C$. The dashed curve shows the proportion of runs achieving an honest signalling equilibrium (i.e., for which $\bar{\epsilon}<\epsilon_{\text {thresh }}$ ). The solid curve shows the average value of $\bar{\rho}$ normalised w.r.t. the maximum observed $\bar{\rho}$ value (std. dev. values were small, varying between $1 \%$ and $3 \%$ of the mean). The inset plot re-plots the solid curve on a log scale. 


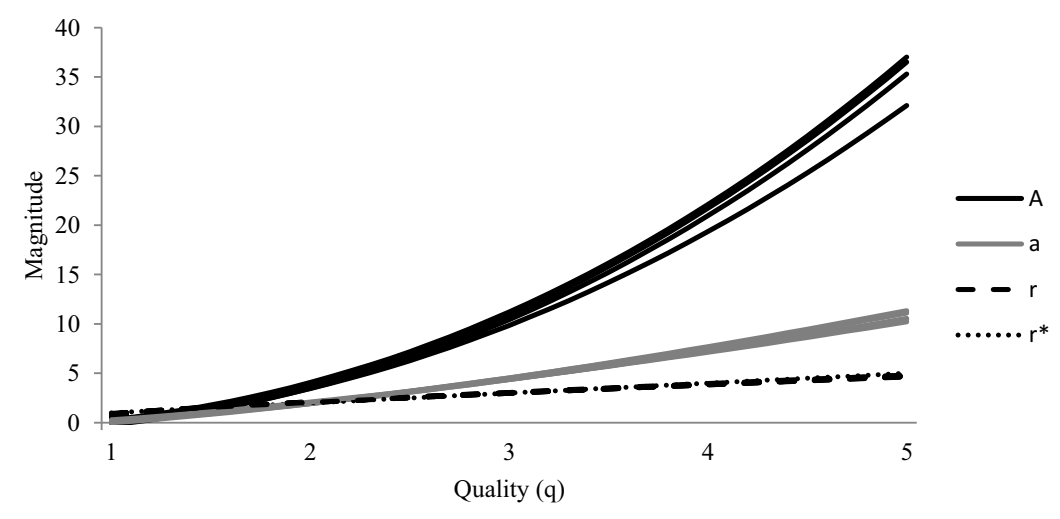

Figure 7: Honest signalling system behaviour for ten distinct scenarios for which $B>C$. Ten evolved signaller mappings, $q \mapsto a$, separate into two bundles, one for scenarios where $B-C=1$ (grey curves, $a$ ) and one for scenarios where $B-C=2$ (solid curves, $A$ ). In each case, receiver strategies produce responses, $r$, that are near optimal (i.e., the dashed response curves lie close to the dotted line, $\left.q \mapsto r^{*}\right)$. The two sets of $\{B, C\}$ scenarios depicted are $\{\{-1,-2\},\{0,-1\},\{1,0\},\{2,1\},\{3,2\}\}$ (grey) and $\{\{0,-2\},\{1,-1\},\{2,0\}$, $\{3,1\},\{4,2\}\}$ (black). Simulation parameters are as per figure 5 . 

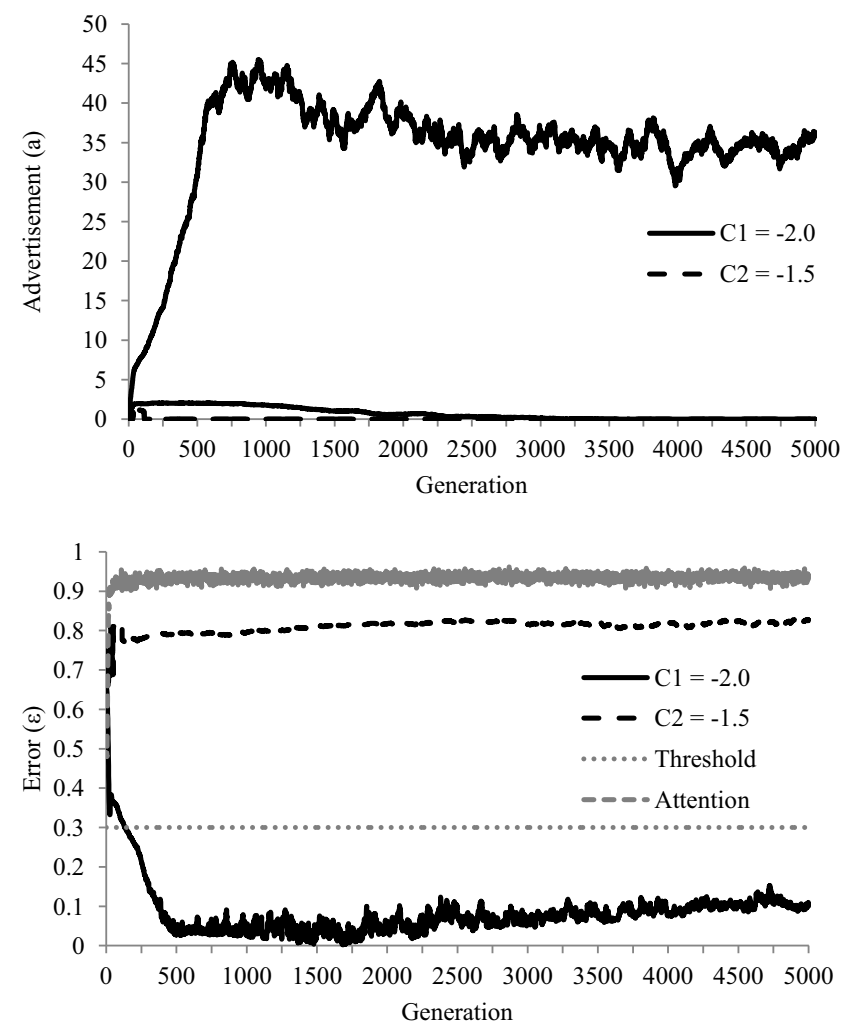

Figure 8: Change in signaller behaviour (upper panel) and receiver behaviour (lower panel) over evolutionary time under conditions that support honest signalling on two competing signalling channels ( $B=0, C_{1}=-2$, solid curves; $B=0, C_{2}=-1.5$, dashed curves). For signallers, a pair of curves for each channel indicate evolutionary change in the magnitude of advertisements $(a)$ given by highest quality and lowest quality signallers employing the mean signaller strategy at each generation during a representative simulation run. For receivers, two curves indicate evolutionary change in the receiver error $(\bar{\epsilon})$ produced by receivers employing the mean receiver strategy for each channel at each generation during the same simulation run. A third curve (grey) indicates the proportion of receivers paying attention to channel 1 . The dotted grey line at $\bar{\epsilon}=\epsilon_{\text {thresh }}=0.3$ indicates the threshold on receiver error that was used to distinguish the attainment of honest signalling from failure to do so. Simulation parameters are as per figure 5 plus $m=0.05$. 

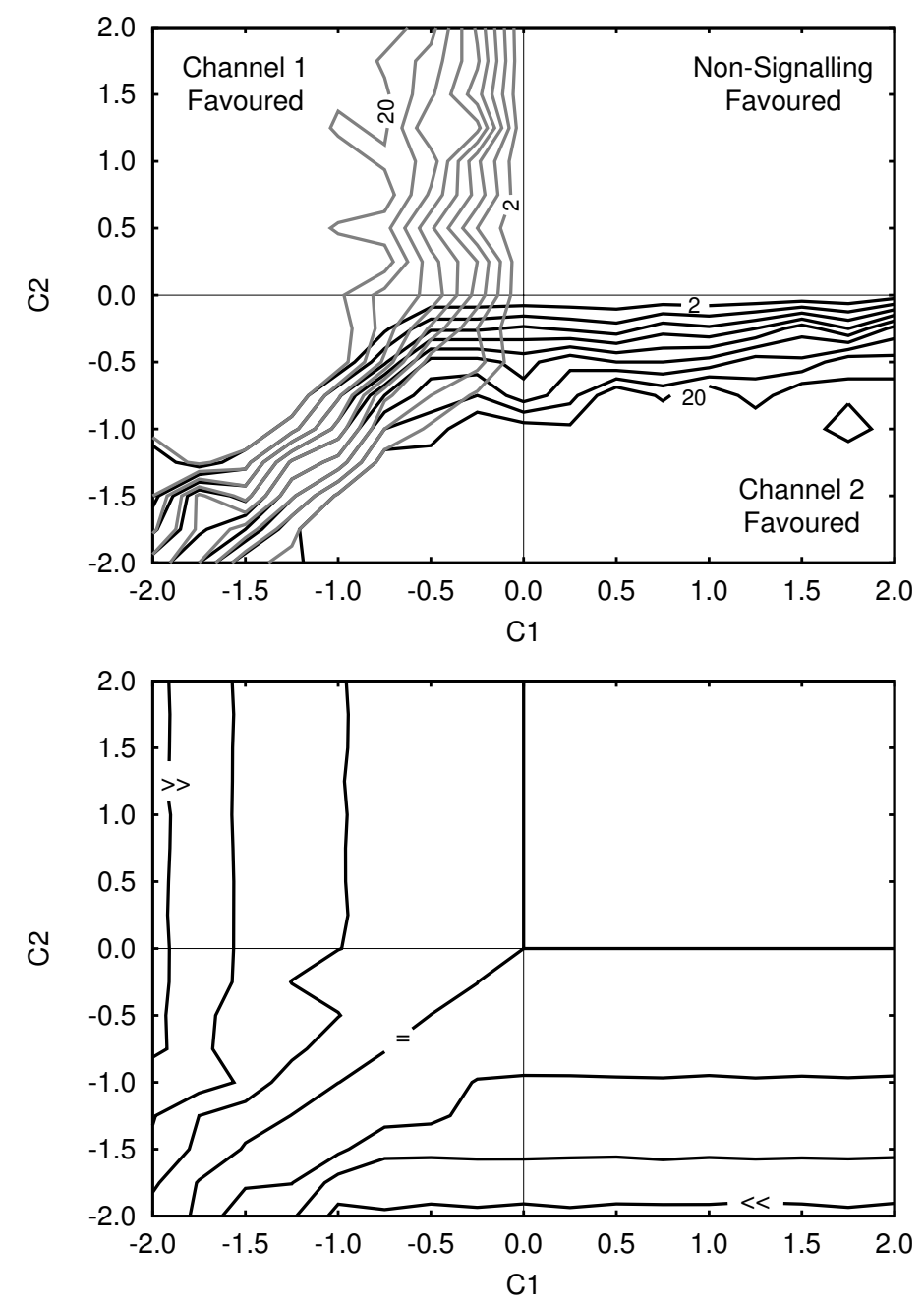

Figure 9: Both panels depict results from the same simulation runs sampling $17 \times 17$ evenly distributed points in the $C_{1} \times C_{2}$ parameter space ( $B=0$ in all cases). Each data point represents an average over 25 simulations runs. Upper panel: the frequency with which honest signalling equilibria are discovered on channel 1 (grey isoclines) and channel 2 (heavy isoclines). Lower panel: the signed difference between the mean signal range on each channel at honest signalling equilibrium, $\bar{\rho}_{1}-\bar{\rho}_{2}$. Isoclines are labeled to indicate scenarios for which $\bar{\rho}_{1} \gg \bar{\rho}_{2}, \bar{\rho}_{1}=\bar{\rho}_{2}$, and $\bar{\rho}_{1} \ll \bar{\rho}_{2}$. (This measure is undefined for the upper right quadrant since no honest signalling equilibria were achieved in this region of parameter space). Parameters as figure 8 . 


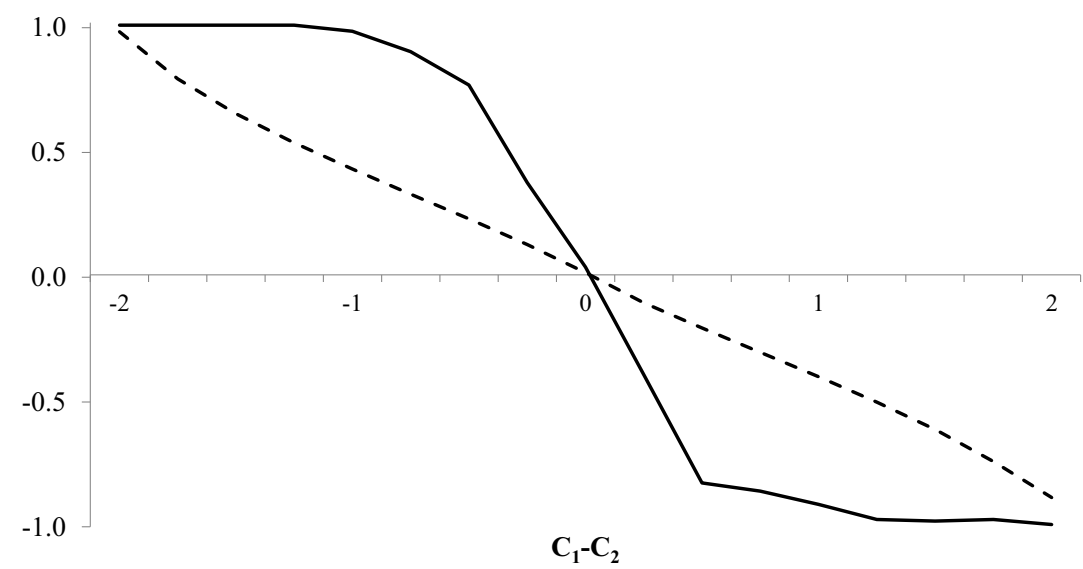

Figure 10: The data from the lower left quadrant of figure 9 (i.e., where both $B>C_{1}$ and $B>C_{2}$ ) are aggregated and replotted against $C_{1}-C_{2}$. The solid curve represents the signed difference between the proportion of simulation runs that achieve honest signalling equilibrium on channel 1 and the proportion that achieved honesty on channel 2 . The dashed curve represents $\bar{\rho}_{1}-\bar{\rho}_{2}$, being the signed difference between the average equilibrium signal range on channels 1 and 2 (std. devs. were small, varying being between $1 \%$ and $3 \%$ of the mean). 


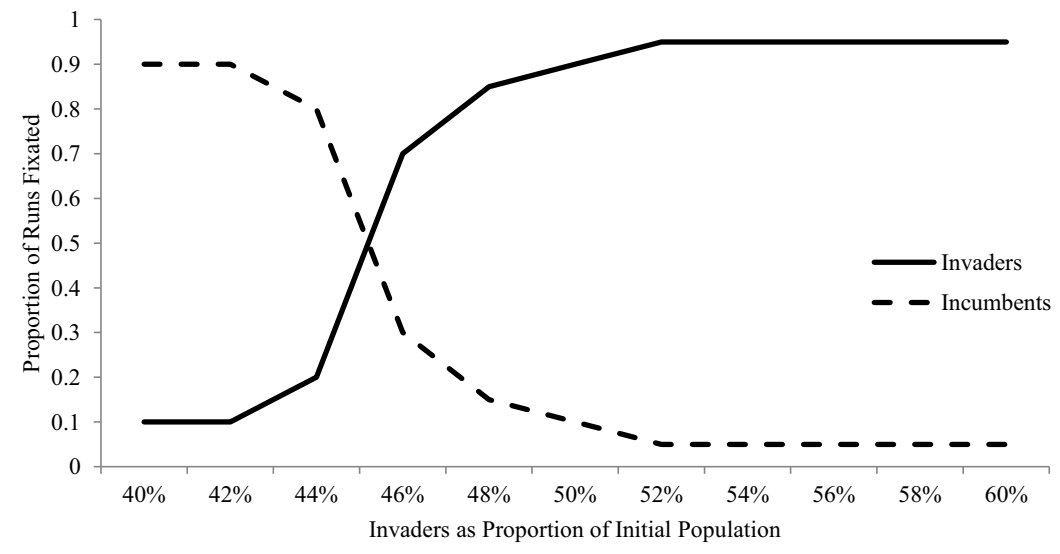

Figure 11: Competition between two established wild-type signalling systems with varying initial frequency in a randomly mixed initial population. The solid curve represents the proportion of $N=20$ simulation runs that fixate on the extravagant invading signalling system $(C=-2)$ after $G=5000$ post-contact generations of evolution. The dashed curve represents the proportion of runs in which the less extravagant incumbent signalling system $(C=-1)$ fixated. $B=0$ for all runs. 